Cerezo, F. (2014). Soporte social en bullying. Análisis de la soledad de la víctima. Revista Electrónica Interuniversitaria de Formación del Profesorado, 17 (1), 123-132.

DOI: http://dx.doi.org/10.6018/reifop.17.1. 198881

\title{
Soporte social en bullying. Análisis de la soledad de la víctima
}

\author{
Fuensanta Cerezo Ramírez \\ Universidad de Murcia
}

\section{Resumen}

Los estudios sobre bullying señalan que estamos ante un problema de relaciones interpersonales donde se configura como factor de riesgo el aislamiento de las víctimas. El objetivo de este estudio es analizar la relación entre victimización en bullying y ascendencia social. Se aplicó el test Bull-S a una muestra de 1478 escolares de ESO (47.8\% chicos y $48.4 \%$ chicas) de entre 11-18 años $(X=14.48 ; d t=1.43$ ) de la Región de Murcia. El 19.4\% estaba implicado en bullying: 129 víctimas. 145 agresores y 13 víctimas-agresoras. El análisis sociométrico confirmó que los implicados son peor valorados que los no implicados y que la condición de víctima está relacionada con rechazo, expectativas de ser rechazado y exclusión. En general, las víctimas del bullying no están más aisladas, sino que son percibidos más negativamente. Estos resultados alertan de la necesidad de mejorar las estrategias de detección e incluir la valoración de las relaciones interpersonales como factor de riesgo o de protección.

\section{Palabras clave}

Bullying; victimización; relaciones interpersonales; rechazo; exclusión.

\section{Social support in bullying. Analysis of the victim loneliness}

\section{Contacto}

Fuensanta Cerezo Ramírez, fcerezo@um.es, Departamento de Psicología Evolutiva y de la Educación. Facultad de Psicología. Universidad de Murcia. Campus de Espinardo. Murcia. 30.100 Murcia. 


\section{Abstract}

Studies about bullying indicate that this is an interpersonal relationships problem and that the victim' isolation is a major risk factor. The main objective is to analyze the relation between being bullying victim and social ascendance. The Bull-S test was completed by 1478 of Secondary Schools ( $47.8 \%$ males, $48.4 \%$ females) from $11-18$ years old $(X=14.48 ; \mathrm{dt}=$ 1.43) from the Region of Murcia (Spain). $19.4 \%$ was implied in bullying: 129 victims, 145 bullies and 13 aggressive-victims. Sociometric analysis confirmed that those pupils that are implied are worse valuated that no implied and being victimized is related to rejection, expectative of being rejected and exclusion. In general, bullying victims are not more isolated, but they are the worst perceived. These results alert from the necessity of improve detection strategies and include interpersonal relationships evaluation as risk or preventive bullying factor.

\section{Key words}

Bullying; victimization; interpersonal relationships; rejection; exclusion.

\section{Introducción}

Las relaciones interpersonales entre los escolares se configuran como una de las principales fuentes de socialización y favorece el desarrollo cognitivo y el ajuste escolar en los adolescentes (Gifford-Smith y Brownell, 2003). Las dificultades en las relaciones entre escolares inciden negativamente, entre otros aspectos, en el desarrollo de la competencia social (Carrasco y Trianes, 2010), la autoestima personal (Boulton, MJ., Smith, PK. \& Cowie, H. 2010; Moreno, Estévez, Pérez y Murgui, 2009) y escolar (Bush, 2005; Martínez, Inglés, Piqueras y Ramos, 2010). Se encuentran diferencias por sexo, así, algunos estudios apuntan que las chicas obtienen puntuaciones más altas en sociabilidad, mientras que los chicos de la misma edad presentan puntuaciones mayores en agresión e inmadurez (Muñoz, Jiménez y Moreno, 2008).

Las relaciones interpersonales vienen definidas por la popularidad de los sujetos, o su estatus. Siguiendo la clasificación de Coie, Dodge \& Kupersmith (1990) en un grupo se pueden distinguir diversos tipos sociométricos o niveles de estatus dentro del aula: el popular o líder, el que es querido por muchos de sus compañeros; el rechazado o impopular, aquel que es rechazado por muchos de sus compañeros; ignorado o aislado, los olvidados, los que no destacan ni por ser populares ni rechazados, los llamados promedio; y por último, los controvertidos, aquellos que reciben bastantes elecciones y a la vez bastantes rechazos. Los estudios ponen de relieve que las experiencias de rechazo en el grupo tiene consecuencias muy negativas, así, se justifica que estos estudiantes presentan un escaso nivel de actividad social, tienen menor autoestima, disfrutan menos con las actividades de clase y se muestran insatisfechos con las relaciones con sus profesores y compañeros (Díaz-Aguado, Martínez y Martín, 2013; García-Bacete, Sureda y Monjas, 2010), lo que favorece que los compañeros se formen una representación negativa de él o ella, ejerciendo refuerzo a sus sentimientos de soledad, resentimiento, ansiedad, depresión, indefensión, y un constante fracaso en sus intentos por participar (Moreno, et al, 2009). Estos sentimientos confluyen en un proceso de conductas de evitación y aislamiento que facilitan la victimización o más bien forman parte de ella (Bush, 2005; Monks, Smith, Naylor, Barter, Ireland \& Coyne, 2009). 
El fenómeno bullying viene a poner de manifiesto las malas relaciones entre los escolares y no sólo por evidenciar un problema de maltrato entre iguales, sino porque en él subyace un conflicto relacional donde algunos se encuentran en clara desventaja social con respecto a otros compañeros (Cerezo y Ato, 2010, Del Rey, Ortega y Feria, 2009; Ortega y MoraMerchán, 2008; Ttofi \& Farrinton, 2008). Aquellos que están implicados en la dinámica bullying presentan niveles de estatus bajos (Cerezo, 2013), es decir, los sujetos con problemas de violencia y/o victimización escolar tienen pocos amigos (Sánchez y Cerezo, 2011), suelen ser menos participativos, con menos competencias sociales (García-Bacete, Sureda y Monjas, 2010), además muestran una deficiente competencia emocional (Sánchez, Ortega y Menesini, 2012) y, por tanto, encuentran dificultades para el manejo de los conflictos e incluso llevar a cabo conductas antisociales (Cerezo y Méndez, 2013).

Aunque ambas caras de la moneda: agresor y víctima obtienen bajos niveles de relaciones sociales (Sánchez, Ortega y Menesini, 2012; Ttofi y Farrington, 2008), las consecuencias son todavía más devastadoras para los víctimas, concretamente, algunos estudios señalan que las experiencias de victimización son buenos predictores de insatisfacción social y de soledad (Kochenderfer-Ladd \& Wardrop, 2001; Salmivalli, 2010) así como de conductas disociales (Bender, D. \& Lösel, F., 2011; Farrington y Ttofi, 2011) y con frecuencia perduran más allá de la escolaridad (Schäfer, et al, 2004; Ttofi, Farrington \& Lösel, 2011).

Aunque ya contamos con algunos estudios que centran sus objetivos en el análisis de las consecuencias de la victimización, todavía no está claramente estudiado qué papel desempaña el estatus social en esta situación. En este estudio nos proponemos responder a las cuestiones: a) ¿Está relacionado el estatus social con la victimización escolar?, b) ¿Están más aislados las víctimas del bullying que el resto de compañeros?, y c) ¿Qué variables de reputación social se relacionan con el rol de víctima?.

\section{Metodología}

\section{Muestra}

La muestra, representativa de los escolares de Educación Secundaria de la Región de Murcia, está formada por 1478 adolescentes ( $47.8 \%$ chicos y $48.4 \%$ chicas) de $11-18$ años (X= $14.48 \mathrm{dt}=1.43)$. Siendo 1191 (80.6\%) Otros, 145 (9.8\%) agresores, 129 (8.7\%) víctimas y 13 víctimas-agresoras (0.9\%).

\section{Procedimiento}

Se solicitó a los centros y a las familias el consentimiento para su participación. Se siguieron los protocolos de voluntariedad, confidencialidad y permiso paterno. Las pruebas se administraron en las aulas de los centros escolares en horario de tutoría con la presencia del profesor o profesora tutor o tutora.

\section{Instrumento}

Se ha empleado el test Bull-S, para la evaluación socio-afectiva del bullying (Cerezo, 2000/2012), basado en la fórmula de la nominación entre iguales o peer nomination. La prueba consta de 15 ítems que se estructuran en 4 dimensiones. Dimensión 1. Valores Sociométricos, con 4 ítems, se pregunta a los alumnos que indiquen con quién de sus compañeros le gusta estar (nominaciones positivas) y con quienes no (nominaciones negativas), se establece un número máximo de 3 nominaciones. Ejemplo de pregunta: "¿A quién elegirías como compañero de grupo?. Dimensión 2. Dinámica agresión y victimización, mediante 6 ítems. Ejemplo de pregunta: “¿Quiénes suelen empezar las peleas?". Esta dimensión facilita la adscripción de sujetos a los diferentes roles, así 
consideramos destacados aquellos sujetos que obtienen, al menos, el $25 \%$ de las nominaciones como agresor, como víctima o como víctima-agresor (cuando destacan en ambos simultáneamente), todos aquellos que no destacan en ninguna de las anteriores conforman el grupo Otros. También indaga sobre características socio-afectivas y relacionales de cada sujeto según la valoración de los compañeros y de uno mismo. Ejemplo de pregunta “¿A quienes se les tiene manía?”; Dimensión 3. Variables situacionales: frecuencia, lugares y forma de las agresiones. Con 2 ítems de elección múltiple ordenada sobre 4 posibilidades. Ejemplo de pregunta: “¿Con qué frecuencia suelen ocurrir las agresiones?". Dimensión 4. Valoración afectiva de las situaciones, con 2 ítems de respuesta escalada en cuatro puntos, indaga sobre la gravedad atribuida y la seguridad percibida en el centro. Ejemplo de pregunta: “¿Te encuentras seguro en el centro escolar?”. La prueba presenta una validez global medida por el alfa de Cronbach de .73 , siendo de .82 para los ítems relacionados con conductas de agresión y de .83 para conductas de victimización. En este estudio hemos utilizamos los datos relativos a la adscripción de los sujetos a los diferentes roles en bullying: Agresor, Víctima, Víctima-Agresor y Otro (Olweus, 1993) y relativos a la primera dimensión: variables Aceptación, Rechazo, Expectativa de ser elegido y Expectativa de ser rechazado, siendo estos datos consistentes para la valoración del estatus social. También se analizan los datos relativos a los de la variable Exclusión. Los análisis sociométricos permiten la clasificación de los sujetos según su Estatus en: 1) Popular o muy elegido, 2) Promedio, 3) Rechazado, 4) Aislado y 5) Controvertido, cuando resulta popular y rechazado a la vez.

\section{Análisis de datos}

Previo al análisis se procedió a la adscripción de los sujetos a los diferentes roles, destacando 129 víctimas en el conjunto de la muestra, representa al $8.7 \%$ del total. Los casos de víctimas-agresores fueron descartados de los análisis. Además, como paso previo a los análisis, se dicotomizó la muestra en: Víctima y no víctima.

\section{Resultados y Discusión}

Roles en Bullying y Estatus sociométrico. Relación entre Estatus social y Victimización en Bullying

Entre los sujetos de la muestra, casi el $20 \%$ se relaciona activamente con el bullying, el 9,8\% como agresores, el $8.7 \%$ como víctimas y casi el $1 \%$ participan de los roles de agresor y de víctima simultáneamente (Véase Tabla 1), en consonancia con otros estudios (Cerezo y Méndez, 2013). En cuanto a la distribución de la muestra por estatus, destaca que la mayoría se encuentran en un nivel promedio (García-Bacete, et al, 2010). Encontramos más sujetos aislados (22.7\%) que rechazados (7.8\%) (Cerezo, 2013; Cerezo y Ato, 2010; Díaz-Aguado, et al, 2013), solamente el $1.8 \%$ resultó popular o muy elegido y no se encontró ningún sujeto controvertido.

La distribución del estatus por No víctima (1) vs Víctima (2) como puede apreciarse en la Tabla 1, indica que ningún alumno víctima es popular, sin embargo, si son la mayoría de los rechazados ( $p>$.001) (García-Bacete, et al 2010; Ortega y Mora-Merchán, 2008). Sin embargo, no encontramos más aislados entre los víctimas que entre los no víctimas $(15.9 \%$ vs 23.6\%), lo que confronta con otros estudios (Monks, et al, 2009; Moreno, et al 2009). pero si más rechazados ( $5.5 \%$ vs $25.8 \%$ ).

En general, el estatus social es más bajo entre los víctimas ( $p>.001)$ aunque el tamaño del efecto es pequeño, es decir, el estatus social tiene un efecto significativo, aunque mínimo sobre la victimización. 
Tabla 1. Distribución de la muestra por sexo, Edad, Rol en bullying y Estatus

1= No Víctima 2= Víctima. Tamaño del efecto en Phi

\begin{tabular}{|c|c|c|c|c|}
\hline Variable & & $\begin{array}{l}\text { Frecuencia } \\
(\mathrm{N})\end{array}$ & $\begin{array}{l}\text { Porcentaje } \\
(\%) \\
\end{array}$ & $\mathrm{X} 2(\mathrm{GL}) ; p$ \\
\hline \multirow[t]{7}{*}{$\operatorname{Sexo}(*)$} & Hombre & 706 & 47.8 & $44,007(2) ; p>.001$ \\
\hline & 1 & 599 & $44 \cdot 9$ & $f=, 173$ \\
\hline & 2 & 99 & 75.0 & \\
\hline & Mujer & 715 & 52.2 & \\
\hline & 1 & 678 & 50.9 & \\
\hline & 2 & 32 & 24.2 & \\
\hline & $11-13$ & 382 & 25.8 & $26,4(21) ; p>.05$ \\
\hline \multirow[t]{2}{*}{ Edad } & $14-15$ & 728 & 49.2 & \\
\hline & $16-18$ & 368 & 25.0 & \\
\hline \multirow[t]{4}{*}{ Bullying } & Otro & 1191 & 80.6 & \\
\hline & Agresor & 145 & 9.8 & \\
\hline & Víctima & 129 & 8.7 & \\
\hline & V-Agresor & 13 & 0.9 & \\
\hline Estatus & Popular & 26 & 1.8 & $75,301(3) ; p>.001$ \\
\hline \multirow[t]{11}{*}{1 vs 2} & 1 & 2 & 1.8 & $f=, 227$ \\
\hline & 2 & 0 & 0 & \\
\hline & Promedio & 1001 & 67.7 & \\
\hline & 1 & 920 & 69.0 & \\
\hline & 2 & 77 & 58.3 & \\
\hline & Aislado & 336 & 22.7 & \\
\hline & 1 & 314 & 23.6 & \\
\hline & 2 & 21 & $15 \cdot 9$ & \\
\hline & Rechazado & 115 & 7.8 & \\
\hline & 1 & 73 & $5 \cdot 5$ & \\
\hline & 2 & 34 & 25.8 & \\
\hline
\end{tabular}

${ }^{*}$ ) Las diferencias con los totales se deben a los casos perdidos y a que la división No Víctima-Víctima deja fuera a los Víctimas-Agresores

Comparaciones entre víctimas y no-víctimas en variables sociométricas.

Se dividió el conjunto de puntuaciones totales de las variables Elegido, Rechazado, Expectativa de ser elegido, Expectativa de ser rechazado y Manía en 2 categorías agrupando los valores: Bajo- Medio como No y Alto y Muy alto como Si, según el rango de cada una de ellas. Una vez dicotomizadas se procedió al análisis de diferencias con Chi- 
Cuadrado (véase Tabla 2). Como se trata de tablas $2 X_{2}$, se consideró como tamaño del efecto el valor de Phi.

$f=$ Tamaño del Efecto para variables dicotómicas

Tabla 2. Tablas de contingencia no víctimas (1) y víctimas (2) en Bullying Con valores dicotomizados: $\mathrm{Si}=$ Alto-Muy Alto; $\mathrm{No}=$ Bajo-Medio

\begin{tabular}{|c|c|c|c|c|c|c|}
\hline \multirow[t]{2}{*}{ Variable } & \multicolumn{6}{|c|}{ Prevalencia \% (N) } \\
\hline & 1 & 2 & $\mathrm{X} 2$ & gl & $p$ & $\mathbf{F}$ \\
\hline Elegido & & & 6,653 & 1 & .010 & 067 \\
\hline No & $91.3(1217)$ & 97.7 (129) & & & & \\
\hline Si & $8.7(116)$ & $2.5(3)$ & & & & \\
\hline Rechazado & & & 45,774 & 1 & .000 & ,177 \\
\hline No & $99,2(1322)$ & $91,7(121)$ & & & & \\
\hline Si & $50.0(11)$ & $50.0(11)$ & & & & \\
\hline Expectativa & & & 22,893 & 1 & .000 & ,129 \\
\hline \multicolumn{7}{|l|}{ Elegido } \\
\hline No & $91,1(1215)$ & $78.0(103)$ & & & & \\
\hline Si & $8.9(118)$ & $22.0(29)$ & & & & \\
\hline Expectativa & & & 39,324 & 1 & .000 & 164 \\
\hline Rechazo & & & & & & \\
\hline No & $94.6(1261)$ & $80.3(106)$ & & & & \\
\hline Si & $5,4(72)$ & $19,7(26)$ & & & & \\
\hline Exclusión & & & 202,885 & 1 & .000 & ,372 \\
\hline No & $98.0(1306)$ & $71,2(94)$ & & & & \\
\hline Si & $2.0(27)$ & $28.8(38)$ & & & & \\
\hline
\end{tabular}

Los análisis con las variables sociométricas indican que: en la variable Ser elegido destacan los No víctimas significativamente, así como en expectativas de ser elegido (Díaz-Aguado, 2013; García-Bacete, et al, 2010), por el contrario, cuando se comparan variables de rechazo los victimas obtienen mayores puntuaciones, en ambos casos los valores de las diferencias se pueden considerar pequeñas (Salmivalli, 2010; Sánchez y Cerezo, 2011). La variable Exclusión presenta mayores valores para las víctimas y el valor de esta diferencia se pude considerar moderado.

Además estos análisis apuntan que las víctimas presentan una alta probabilidad de ser más rechazadas que el resto de la muestra. Del mismo modo, las expectativas de ser rechazado pueden considerarse como un elemento asociado a la victimización (Sánchez, Ortega, y Menesini, 2012). A esto hay que añadir que es más probable que las víctimas sean excluidos frente a los no víctimas (Kochenderfer-Ladd \& Wardrop, 2001). En cuanto al sexo, encontramos que los hombres tienen mayor probabilidad de ser víctimas que las mujeres ( $p>$.001), coincidiendo con otros estudios (Muñoz, Jiménez y Moreno, 2008).

Reputación social y victimización

Para responder a la cuestión sobre qué variables de reputación social se relacionan con el rol de víctima se realizó un análisis de ANOVA, análisis de varianza de diferencias entre las puntuaciones medias obtenidas en las variables de aceptación, rechazo, expectativa de aceptación, expectativa de rechazo y exclusión. Los resultados pueden apreciarse en la Tabla 3 
Los valores de las medias y dt tomados del análisis $t$

$d=$ Tamaño del Efecto. Calculado con dt combinada

Tabla 3. Análisis de varianza en variables sociométricas Anovas entre: No víctimas (1) y Víctimas (2) en Bullying

\begin{tabular}{|c|c|c|c|c|c|}
\hline Variables & $x$ & $\mathrm{dt}$ & $F(1 ; 1463)$ & $p$ & d \\
\hline Elegido & & & 34,136 & .000 & -1.182 \\
\hline 1 & 4.98 & 3.896 & & & \\
\hline 2 & 2.95 & 2.922 & & & \\
\hline Rechazado & & & 142,929 & .000 & 3.145 \\
\hline 1 & 3.72 & 5.177 & & & \\
\hline 2 & 10.01 & 10.233 & & & \\
\hline Expectativa & & & 8,351 & .004 & .435 \\
\hline Elegido & 4.73 & 3.332 & & & \\
\hline 1 & 3.86 & 2.910 & & & \\
\hline \multicolumn{6}{|l|}{2} \\
\hline Expectativa & & & 47,632 & .000 & 1.001 \\
\hline Rechazo & 4.03 & 4.389 & & & \\
\hline 1 & 7.13 & 6.392 & & & \\
\hline \multicolumn{6}{|l|}{2} \\
\hline Exclusión & & & 406,503 & .000 & 4.515 \\
\hline 1 & 6.15 & $9 \cdot 344$ & & & \\
\hline 2 & 25.42 & 18.375 & & & \\
\hline
\end{tabular}

En general, los análisis muestran una mayor incidencia de las variables de reputación negativa entre las víctimas, así destacan en Rechazo, Expectativa de Rechazo y en Exclusión (Buhs, 2005; García-Bacete, et al, 2010; Moreno, et al, 2009; Muñoz, et al, 2008), siendo en todos los casos, el valor del Tamaño del Efecto grande (mayor de 1), mientras que las diferencias en las variables Ser elegido y Expectativa de ser elegido no resultan significativas.

\section{Conclusiones}

En cuanto al nivel de Estatus social, entre los víctimas del bullying no se confirma que destaquen por ser aisladas, sino por ser rechazadas. El análisis de las variables sociométricas confirma que la victimización se asocia significativamente con reputación social negativa ya que son más excluidos y rechazados. Es de mencionar que las víctimas consideran que van a ser más elegidos cuando en realidad no lo son, lo que puede indicar un desajuste de su auto-percepción social, sin embargo y paralelamente si reconocen que serán más rechazados, lo que indica que la percepción negativa está más ajustada a la realidad. Esto puede suponer una sobrevaloración de las relaciones afectivas positivas frente a un sentimiento de exclusión social. Aspecto que precisaría de futuras investigaciones.

El trabajo también indica que el bullying está más asociado a los varones que a las mujeres, tanto como agresores como víctimas.

Así pues no se confirma que las víctimas del bullying estén más aisladas que el promedio del grupo, por lo que cabe considerar que el grupo ejerce sobre ellas un tipo de violencia social: la exclusión, que todavía la sitúan en mayor indefensión. 


\section{Bibliografía}

Bender, D. \& Lösel, F. (2011). Bullying at school as a predictor of delinquency, violence and other anti-social behavior in adulthood. Criminal Behaviour and Mental Health, 21 (99106)

Buhs, E. S. (2005). Peer rejection, negative peer treatment, and school adjustment: Selfconcept and classroom engagement as mediating processes. Journal of School Psychology, 43, 407-424

Boulton, MJ., Smith, PK. \& Cowie, H. (2010). Short-Term Longitudinal Relationships Between Children's Peer Victimization/Bullying Experiences and Self-Perceptions Evidence for Reciprocity. School Psychology International, 31(3), 296-311

Carrasco, C. y Trianes, M. V. (2010). Clima social, prosocialidad y violencia como predictores de inadaptación escolar en primaria. European Journal of Education and Psychology, 3 (2), 229-242

Cerezo, F. (2013). Diferencias en estatus social entre roles en bullying. Un análisis sociométrico. Bordón, 62(2) en prensa.

Cerezo, F. \& Ato, M. (2010). Social status, gender, classroom climate and bullying among adolescents pupils. Anales de Psicología, 26 (1), 137-144.

Cerezo, F., y Méndez, I. (2013). Agresores en bullying y conductas antisociales. European Journal of Investigation in Health, Psychology and Education,3 (1), 5-14

Del Rey, R., Ortega, R. y Feria, I. (2009). Convivencia escolar: fortaleza de la comunidad educativa y protección ante la conflictividad escolar. Revista Interuniversitaria de Formación del Profesorado, 66 (23,3), 159-180

Díaz-Aguado, M. J.; Martínez, R. y Martín, J. (2013) El acoso escolar en España. Prevalencia, papeles adoptados por todo el grupo y características a las que atribuyen la victimización. Revista de Educación, DOI:10.4438/1988-592X-RE-2011-362-164.

Farrington, D. P. \& Ttofi, M. M. (2011). Bullying As a predictor of offending violence and later outcomes. Criminal Behaviour and Mental Health, 21 (90-98)

García-Bacete, F. J., Sureda, I. \& Monjas, I. (2010). El rechazo entre iguales en la educación primaria: Una panorámica general. Anales de Psicología, 26(1), 123-136.

Gifford-Smith, M. E. \& Brownell, C. A. (2003). Childhood peer relationships: social acceptance, friendships, and peer networks. Journal of School Psychology, 41. 235284

Hunter, S.C., Mora-Merchán, J. y Ortega, R. (2004). The Long-Term effects of Coping Stgrategies Use in Victims of Bullying. The Spanish Journ al of Psychology, 7(1), 3-12

Kochenderfer-Ladd, B. \& Wardrop, JL. (2001). Chronicity and instability of children's peer victimitation experiencies as predictors of loneliness and social satisfaction trayectories. Child Development, 72(1), 134-151.

Martínez, E., Inglés, CJ, Piqueras, J. y Ramos, V. (2010). The importance of friends and parents in health and academic performance. Electronic Journal of Research in Educational Psychology, 8(1), 111-138 
Monks, C. P.; Smith, P. K.; Naylor, P.; Barter, C.; Ireland, J. L. \& Coyne, I. (2009). Bullying in different contexts: Commonalities, differences and the role of the theory. Aggression and Violent Behavior, 14, 146-156.

Moreno, D., Estévez, E., Pérez, S., Murgui, G., (2009). Reputación social y violencia relacional en adolescentes: el rol de la soledad, la autoestima y la satisfacción vital. Psicothema, 21 (4), 537-542

Muñoz, M. V., Jiménez, I. Y Moreno, M. C. (2008). Reputación conductual y género en la adolescencia. Anales de Psicología, 24(2), 334-340.

Ortega, R., \& Mora-Merchán, J. (2008). Las redes de iguales y el fenómeno del acoso escolar: explorando el esquema dominio-sumisión. Infancia y Aprendizaje, 31 (4), 515528.

Salmivalli, C. (2010). Bullying and the peer group: A review. Aggression and Violent Behavior. $15,112-120$.

Schäfer, M., Korn, S., Smith, P.K., Hunter, S.C., Mora-Merchán, J. A., Singer, M. \& van der Meulen, K. (2004). Lonely in the crowd: Recollections of bullying. British Journal of Developmental Psychology, 22, 379-394

Sánchez, C. \& Cerezo, F. (2011). Estatus social de los sujetos implicados en bullying. Elementos para la mejora de la convivencia en el aula. REOP, 22(2), 137-149

Sánchez, V., Ortega, R. y Menesini, E. (2012). La competencia emocional de agresores y víctimas en bullying. Anales de Psicología, 28(1),71-82

Ttofi, M M., \& Farrington, D P. (2008) Short-term and long-term effects of bullying and the importance of Defiance Theory in explanation and prevention. Victims and Offenders 3: 289-317.

Ttofi, M M., Farrington, D P. \& Lösel, F. (2011). Health consequences of school bullying. Journal of Aggression, Conflict and Peace Research, 3(2), 60-62. 


\section{Autores}

Fuensanta Cerezo Ramírez

Profesora Titular de Psicología de la Educación de la Universidad de Murcia. Imparte docencia en los títulos de grado y posgrado en las titulaciones de Educación, Psicología y Criminología. Sus investigaciones están en la línea de: la violencia escolar su conceptualización, evaluación, prevención e intervención; la adolescencia y sus problemas y en la formación del profesorado. Ha trabajado como profesor colaborador en diversas universidades españolas y extranjeras (europeas y americanas) dentro de la línea de la Psicología de le Educación y específicamente con temas relacionado con la violencia escolar. Participa como asesora con el Instituto de Servicios Sociales de la Región de Murcia para la elaboración de protocolos de evaluación de niños maltratados, y otras asociaciones relacionadas con la infancia y el bullying 